\title{
A myriad of electrocardiographic findings associated with digoxin use
}

Andie Hartanto Djohan ${ }^{1}$, MBBS, MRCP, Ching-Hui Sia ${ }^{1,2}$, MBBS, MRCP, Devinder Singh ${ }^{1,2}$, MD, MRCP, Weiqin $\underline{\operatorname{Lin}}^{1,2}$, MBBS, MRCP, William Kok-Fai $\underline{K o n g}^{1,2}$, MD, Kian-Keong ${\underline{P o h^{1}}}^{1,2}$, MBBChir, FACC

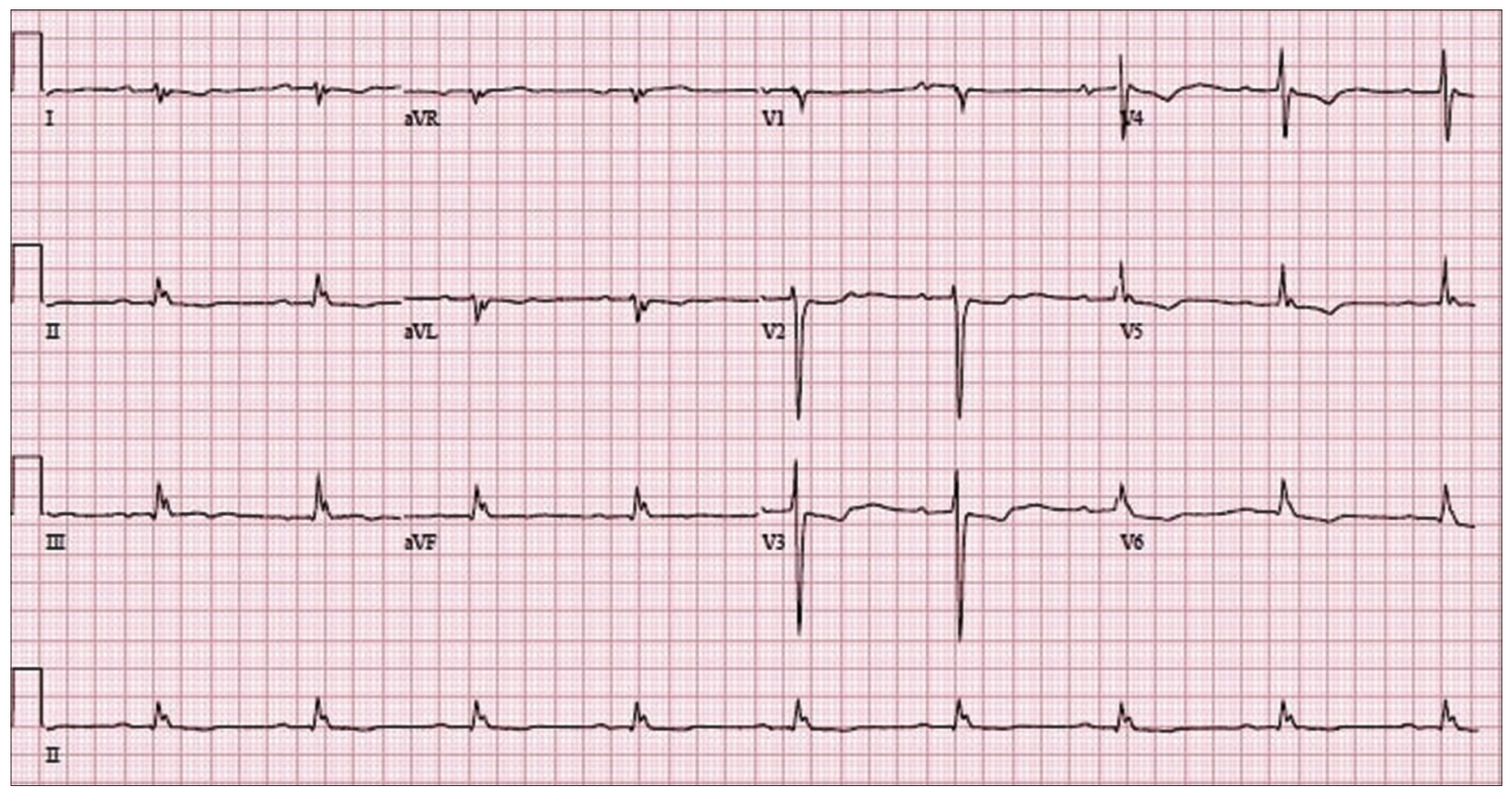

Fig. 1 The patient's 12-lead ECG on admission.

\section{CASE 1}

\section{CLINICAL PRESENTATION}

A 77-year-old woman with known ischaemic cardiomyopathy was admitted for an episode of acute decompensated heart failure (HF) precipitated by non-compliance to medications. She was on digoxin 125 mcg once daily as part of HF treatment. What does her admission electrocardiogram (ECG) show (Fig. 1)?

\section{ECG INTERPRETATION}

The ECG in Fig. 1 shows a baseline sinus rhythm with a heart rate of 60 beats per minute (bpm). There is evidence of digoxin-induced ECG change (reverse-tick ST segment) that is most easily appreciated in leads V2-V5. This is more accurately described as a biphasic T wave with an initial negative and terminal positive deflection, and is most commonly seen in leads with a dominant $R$ wave (V4-V6). There is evidence of PR prolongation (first-degree atrioventricular [AV] block) due to the effect of digoxin in increasing vagal tone.

\section{CLINICAL COURSE}

The patient's presenting chest radiograph (Fig. 2) showed features consistent with congestive HF, such as alveolar oedema (bat's wing opacities), Kerley B lines, cardiomegaly, dilated upper lobe pulmonary vessels and pleural effusion. She was treated with intravenous (IV) furosemide $40 \mathrm{mg}$ twice daily and her symptoms improved remarkably.

It is important to determine the underlying cause of decompensation. Common causes include ischaemia (myocardial infarction), tachyarrhythmias (atrial fibrillation with rapid ventricular response, supraventricular or ventricular tachycardias), bradyarrhythmias (heart blocks), sepsis, and non-compliance to medications, fluid and dietary restrictions. In Case 1, the decompensation was due to poor compliance to medications and fluid restrictions. Every possible effort should be made to address the underlying cause to reduce future readmissions.

${ }^{1}$ Department of Cardiology, National University Heart Centre Singapore, ${ }^{2}$ Yong Loo Lin School of Medicine, National University of Singapore, Singapore 


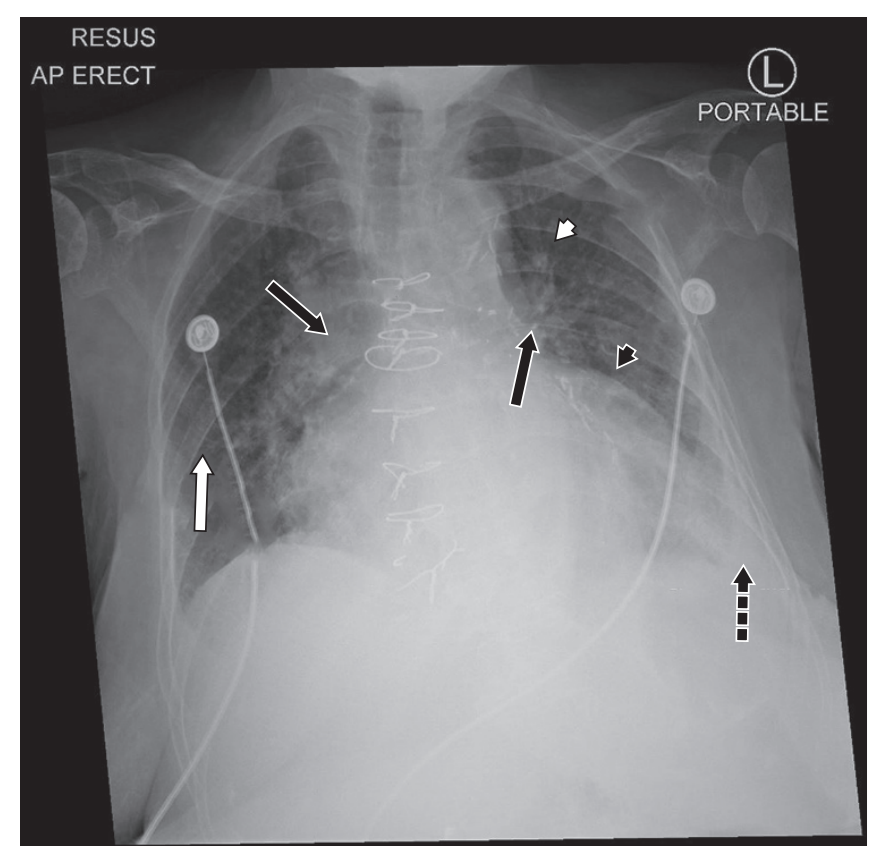

Fig. 2 Chest radiograph of the patient on presentation shows the features of congestive heart failure: alveolar oedema (black arrows), interstitial or Kerley B lines (white arrow), cardiomegaly (black arrowhead), dilated upper lobe vessels (white arrowhead) and pleural effusion (dotted arrow).

\section{CASE 2}

\section{CLINICAL PRESENTATION}

A 68-year-old woman was flown into Singapore via emergency air ambulance for shortness of breath of two days' duration. She had a history of pulmonary arterial hypertension secondary to congenital right pulmonary arteriovenous malformation, complicated by recurrent episodes of HF. The patient was diagnosed with cardiogenic shock and admitted into the coronary care unit (CCU). Her acute presentation was complicated by acute kidney injury secondary to cardiorenal syndrome and hyperkalaemia. Relevant cardiac medications included bosentan $62.5 \mathrm{mg}$ twice daily, digoxin $62.5 \mathrm{mcg}$ once daily, tadalafil $20 \mathrm{mg}$ once daily and furosemide $40 \mathrm{mg}$ twice daily.

During her stay in the $\mathrm{CCU}$, she developed an acute episode of confusion. Continuous ECG monitoring showed multiple arrhythmias, as captured on 12-lead ECGs (Figs. 3a-c), although her electrolyte levels (potassium, calcium, magnesium) were within normal limits. What do the ECGs show (Figs. 3a-c)?

\section{ECG INTERPRETATION}

The ECG in Fig. 3a shows atrial fibrillation with an irregularly irregular R-R interval. There is no ' $\mathrm{p}$ ' wave. Atrial fibrillation is the patient's baseline ECG rhythm. Fig. 3b shows frequent ventricular ectopic beats with an initial bigeminy pattern. There is narrow complex QRS (black arrowhead) followed by wide complex QRS (white arrowhead). The third beat has a preceding ' $p$ ' wave (white arrow). Subsequent wide complex QRS with alternating beat-to-beat frontal plane axis (black arrows) is documented on lead II. This is a classic instance of bidirectional ventricular tachycardia, an arrhythmia associated with digoxin toxicity, but is also seen in familial catecholaminergic polymorphic tachycardia.
The ECG in Fig. 3c shows a regular broad complex rhythm at a rate of $60 \mathrm{bpm}$. The QRS morphology is consistent with that of left bundle branch block. Accelerated idioventricular rhythm with capture beat (arrowhead) and fusion beat (arrow) is seen.

\section{CLINICAL COURSE}

The patient underwent an episode of cardiorenal syndrome secondary to acute decompensated $\mathrm{HF}$, with serum creatinine rising from $63 \mu \mathrm{mol} / \mathrm{L}$ to $242 \mu \mathrm{mol} / \mathrm{L}$. Her symptoms and ECG changes in the presence of worsening renal function were suggestive of digoxin toxicity. A serum digoxin level of $3.6 \mu \mathrm{g} / \mathrm{L}$ confirmed the diagnosis of digoxin toxicity. Digoxin-specific antibody fragments were administered as a reversal agent. There was resolution of arrhythmias, with a corresponding normalised serum digoxin level of $1.3 \mu \mathrm{g} / \mathrm{L}$.

\section{DISCUSSION}

Digoxin is a drug used in treatment of HF as well as for heart rate control in atrial fibrillation. The results of the Digitalis Investigation Group (DIG)(1) trial, published in 1997, were central in establishing the place of digoxin in the treatment of HF relative to other established pharmacological therapies. In this trial, patients with left ventricular ejection fraction $\leq 45 \%$ and in normal sinus rhythm were randomised to receive either digoxin or a placebo, with a background of concomitant treatment using angiotensinconverting enzyme (ACE) inhibitors and diuretics. $80 \%$ of the patients had New York Heart Association Class II-III HF. The results showed that digoxin has no effect on the primary outcome of all-cause mortality. However, the rate of hospitalisation was reduced for the group of patients treated with digoxin, compared to those treated with placebo.

Under the 2016 European Society of Cardiology Guidelines ${ }^{(2)}$ for the diagnosis and treatment of acute and chronic HF, digoxin is currently the fourth-line drug in the management of HF to reduce the risk of both HF-related and all-cause hospitalisation. Similarly, the 2013 American College of Cardiology Guidelines ${ }^{(3)}$ for the management of HF mentions that digoxin may be beneficial for symptomatic improvement of HF patients on top of neurohormonal treatment such as ACE inhibitors. These statements establish digoxin's place as adjunctive pharmacological therapy in the management of HF, largely due to its narrow therapeutic index, and its lack of mortality benefit for HF patients. ${ }^{(4,5)}$

\section{Pharmacology and digoxin-related ECG changes}

Pharmacologically, digoxin has a dual mechanism of action through its inhibition of the cardiac myofibril $\mathrm{Na}^{+}-\mathrm{K}^{+}$ATPase. ${ }^{(6)}$ This leads to increased intracellular $\mathrm{Ca}^{2+}$ concentrations ${ }^{(4,6)}$ and in turn increased inotropy, which may summate to trigger repetitive electrical impulses. It is highly likely that this is the pathophysiological basis of most digoxin-induced tachycardias. ${ }^{(6)}$

Beside its effects on inotropy and automaticity, digoxin also exerts parasympathomimetic actions by increasing vagal tone to both the sinoatrial and $\mathrm{AV}$ nodes, manifesting as a reduction in heart rate and slowing conduction through the AV node. ${ }^{(6)}$ Due its dual action, the classic hallmarks of digoxin toxicity are enhanced 

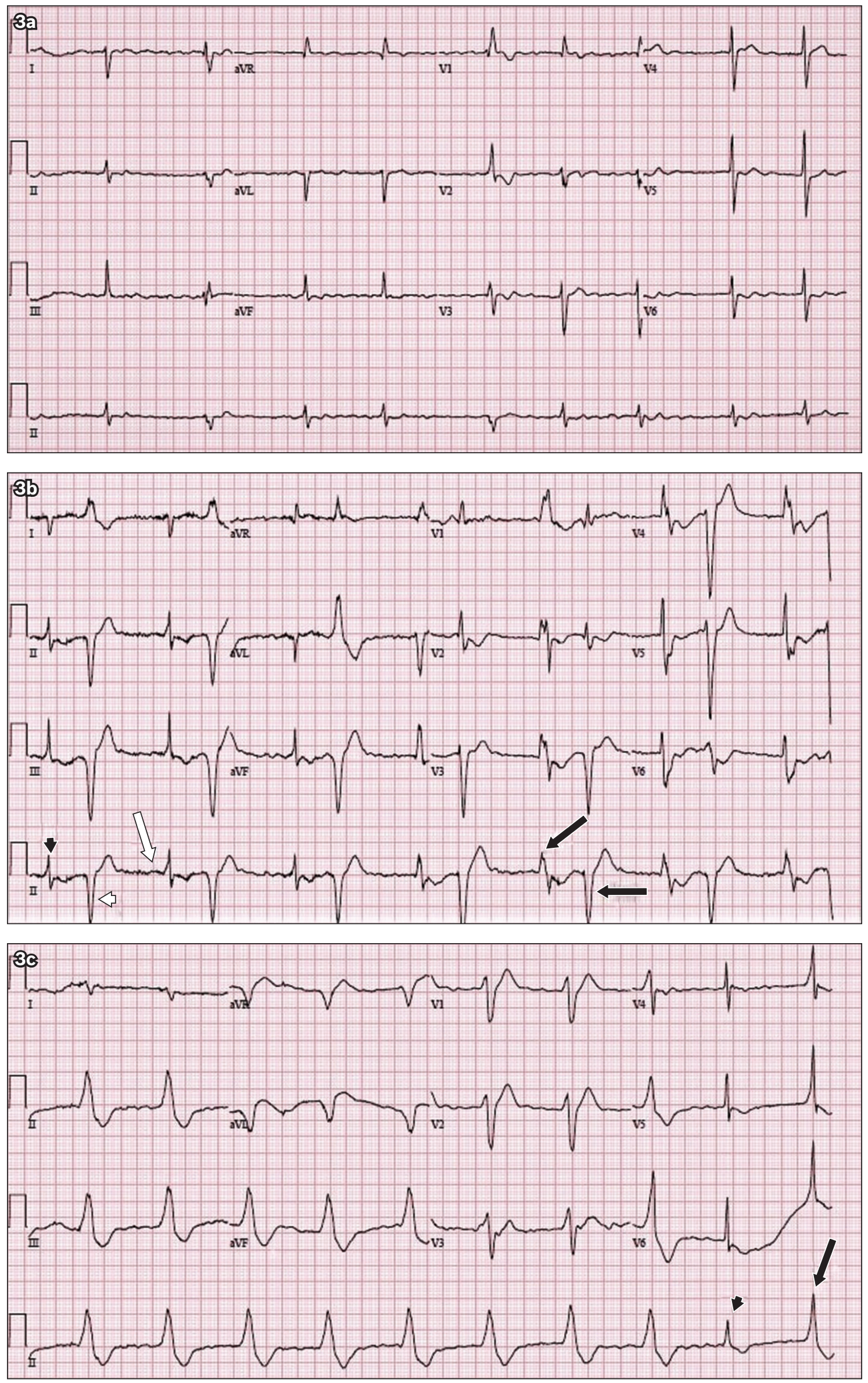

Fig. 3 12-lead ECG shows (a) atrial fibrillation at baseline; and (b) frequent ventricular ectopic beats with an initial bigeminy pattern during acute confusional state. (c) ECG during acute confusion shows further arrhythmia. 
automaticity and impaired conduction. ${ }^{(7)}$ Hence, arrhythmias that are commonly associated with digoxin toxicity include frequent premature ventricular contractions; non-paroxysmal junctional tachycardia; atrial tachycardia with heart block; and as seen in Case 2, atrial fibrillation with regularised ventricular rate due to AV block; as well as bidirectional ventricular tachycardia. (4,7) $^{(4)}$

As opposed to the arrhythmias secondary to digoxin toxicity, therapeutic levels of digoxin cause ECG changes known as the 'digoxin effect', as seen in Case 1. This simply indicates that the patient is on digoxin and is not a marker of toxicity. While the 'reverse tick' pattern in V4-V6 is the most commonly described ECG change, it is more appropriately described as a biphasic $\mathrm{T}$ wave with an initial negative and terminal positive deflection (Fig. 1). These changes are due to the effect of digoxin in reducing the atrial and ventricular refractory periods, causing secondary repolarisation abnormalities that affect the ST segment, T waves and $U$ waves. A shortened QT interval may also be observed as a result of the shortened ventricular refractory period, ${ }^{(8)}$ and a first-degree heart block may be observed due to increased vagal effects on the AV node. ${ }^{(8)}$

\section{Serum digoxin levels and mortality}

The overall incidence of digoxin toxicity was $2 \%$ over three years in the DIG trial. ${ }^{(5)}$ Serum digoxin levels of $>2.0 \mathrm{ng} / \mathrm{mL}$ are usually considered toxic. However, a post-hoc analysis of the DIG trial done by Rathore et $\mathrm{al}^{(9)}$ and Lopes et $\mathrm{al}^{\left({ }^{(10)}\right.}$ demonstrate that serum digoxin concentrations (SDCs) of $\geq 1.2 \mathrm{ng} / \mathrm{mL}$ are associated with increased mortality. Rathore's study ${ }^{(9)}$ shows that an SDC of $0.5-0.8 \mathrm{ng} / \mathrm{mL}$ is the likely optimal therapeutic range for $\mathrm{HF}$ with left ventricular dysfunction. The importance of monitoring SDC is recognised by the National Heart Foundation of Australia and the Cardiac Society of Australia and New Zealand, ${ }^{(11)}$ which recommended that SDC be kept at $<1.2 \mathrm{ng} / \mathrm{mL}$.

Digoxin toxicity can occur in both acute (e.g. overdose) and chronic settings. With reference to Case 2 , digoxin toxicity may occur in patients taking recommended doses $(0.125-0.25 \mathrm{mg}$ once daily) when there is an additional precipitating factor such as hypokalaemia, hypomagnesaemia, hypothyroidism, acute kidney injury and chronic kidney disease. ${ }^{(7)}$ Conditions that predispose a patient to digoxin toxicity are listed in Box 1. Besides cardiac manifestations, symptoms of digoxin toxicity may manifest in other organ systems as confusion, gastrointestinal disturbance, visual blurring and altered coloured vision (Box 2). ${ }^{(4)}$ Electrolyte abnormalities can also result in ECG changes that may occur concomitantly with digoxin-related ECG changes. ${ }^{(12)}$

Besides concomitant disease states, drug-drug interactions may increase SDCs that risk toxicity. Digoxin is a substrate for p-glycoprotein, and inhibition by several known drugs (amiodarone, verapamil, clarithromycin, erythromycin and quinidine) has been implicated in resultant increases in SDCs, as they increase gastrointestinal absorption and reduce renal clearance. ${ }^{(13,14)}$

\section{Recognition and treatment of digoxin toxicity}

Hyperkalaemia in the setting of digoxin blockade of $\mathrm{Na}^{+}-\mathrm{K}^{+}$ ATPase is suggestive of toxic serum concentrations of digoxin
Box 1. Predisposing conditions for digoxin toxicity: $:^{(4,13,14)}$

- Decreased excretion (e.g. renal disease)

- Hypothyroidism

- Drug interaction with amiodarone, verapamil, clarithromycin, erythromycin or quinidine

- Increased myocardial sensitivity

- Myocarditis

- Myocardial ischaemia

- Electrolyte disturbances (hypokalaemia, hypomagnesaemia,

hypernatraemia, acidosis)

- Catecholamines

- Immediate postoperative period after cardiac surgery

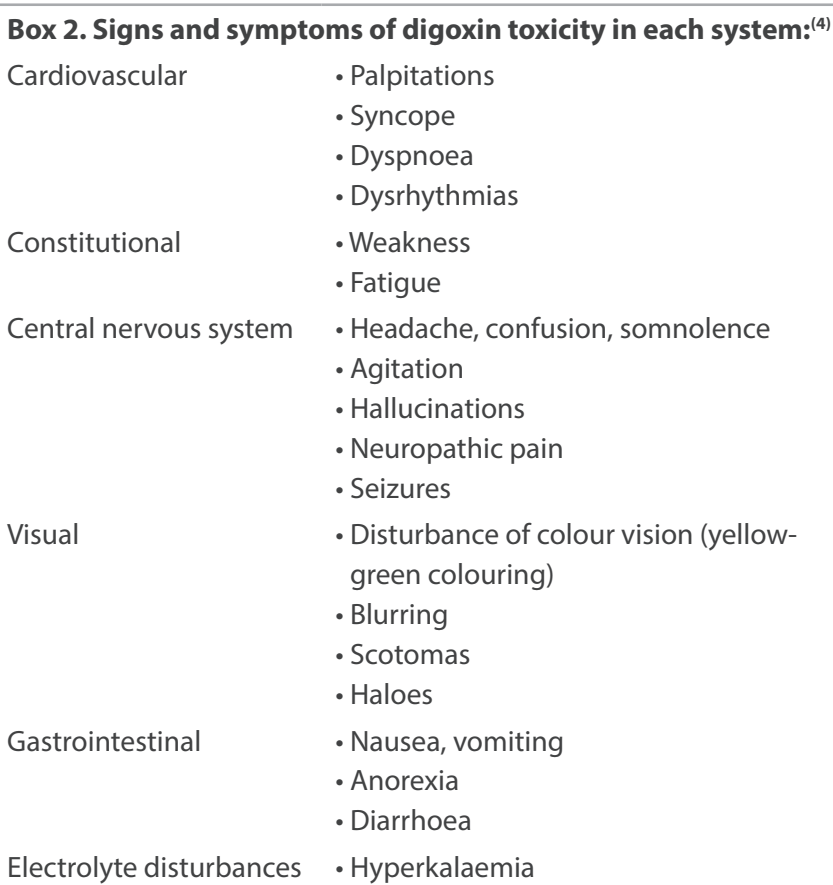

and requires emergency measures. ${ }^{(6)}$ The treatment of digoxin toxicity depends on its clinical manifestations and the presence of haemodynamic instability. Symptomatic bradycardia secondary to excessive AV node blockade may be treated with IV atropine. Higher degrees of AV nodal block may require the initiation of cardiac pacing, and ventricular ectopic beats or arrhythmias may be treated with IV lignocaine. ${ }^{(4)}$ However, the first-line treatment for dysrhythmias that are potentially life-threatening or haemodynamically unstable is IV antidigoxin antibody fragments. Besides hyperkalaemia, other indications for antidigoxin antibody includes acute ingestion of $0.3 \mathrm{mg} / \mathrm{kg}$ of digoxin, serum levels exceeding $5 \mathrm{ng} / \mathrm{mL}$ and rapidly progressive clinical manifestations of digoxin toxicity. ${ }^{(4)}$

Lastly, acute kidney injury, hyperkalaemia and resultant digoxin toxicity may represent differing severity along a spectrum of complications. While haemodialysis may be the treatment of choice for acute kidney injury and hyperkalaemia under the right clinical circumstance, the highly tissue-bound nature of digoxin renders it poorly dialysable, although it is renally cleared in normal physiological processes ${ }^{(6)}$ Hence, caution should be exercised in prescribing it to patients with acute and chronic kidney disease. 
In conclusion, the signs and symptoms of digoxin toxicity are often nonspecific. However, prompt recognition of characteristic ECG changes and having a high index of suspicion will help in diagnosis and treatment, minimising potential complications from an otherwise safe and commonly prescribed drug.

ABSTRACT Digoxin is a commonly prescribed drug in the management of heart failure and atrial fibrillation. Despite its widespread use, most clinicians have little experience with recognising clinical signs and symptoms that might suggest a potentially lethal drug toxicity. We herein describe two cases with specific reference to the electrocardiographic changes induced by digoxin and discuss the predisposing factors for toxicity, recognition of possible toxicity and treatment approaches.

Keywords: digoxin, ECG, toxicity

\section{REFERENCES}

1. Digitalis Investigation Group. The effect of digoxin on mortality and morbidity in patients with heart failure. N Engl J Med 1997; 336:525-33.

2. Ponikowski P, Voors AA, Anker SD, et al; ESC Scientific Document Group. 2016 ESC Guidelines for the diagnosis and treatment of acute and chronic heart failure: the Task Force for the diagnosis and treatment of acute and chronic heart failure of the European Society of Cardiology (ESC) developed with the special contribution of the Heart Failure Association (HFA) of the ESC. Eur Heart J 2016; 37:2129-200.

3. Yancy CW, Jessup M, Bozkurt B, et al; American College of Cardiology
Foundation; American Heart Association Task Force on Practice Guidelines. 2013 ACCF/AHA guideline for the management of heart failure: a report of the American College of Cardiology Foundation/American Heart Association Task Force on Practice Guidelines. J Am Coll Cardiol 2013; 62:e147-239.

4. Thacker D, Sharma J. Digoxin toxicity. Clin Pediatr (Phila) 2007; 46:276-9.

5. Rich MW, McSherry F, Williford WO, Yusuf S; Digitalis Investigation Group. Effect of age on mortality, hospitalizations and response to digoxin in patients with heart failure: the DIG Study. J Am Coll Cardiol 2001; 38:806-13.

6. Bauman JL, Didomenico RJ, Galanter W. Mechanisms, manifestations, and management of digoxin toxicity in the modern era. Am J Cardiovasc Drugs 2006; 6:77-86.

7. Raja Rao MP, Panduranga P, Sulaiman K, Al-Jufaili M. Digoxin toxicity with normal digoxin and serum potassium levels: beware of magnesium, the hidden malefactor. J Emerg Med 2013; 45:e31-4.

8. Burns E. Digoxin Effect. In: Life in the Fastlane [online]. Available at: https:// litfl.com/digoxin-effect-ecg-library/. Accessed November 1, 2019.

9. Rathore SS, Curtis JP, Wang Y, Bristow MR, Krumholz HM. Association of serum digoxin concentration and outcomes in patients with heart failure. JAMA 2003; 289:871-8.

10. Lopes RD, Rordorf R, De Ferrari GM, et al; ARISTOTLE Committees and Investigators. Digoxin and mortality in patients with atrial fibrillation. J Am Coll Cardiol 2018; 71:1063-74.

11. NHFA CSANZ Atrial Fibrillation Guideline Working Group, Brieger D, Amerena J, et al. National Heart Foundation of Australia and the Cardiac Society of Australia and New Zealand: Australian Clinical Guidelines for the diagnosis and management of atrial fibrillation 2018. Heart Lung Circ 2018; 27:1209-66.

12. Kuntjoro I, Teo SG, Poh KK. Abnormal ECGs secondary to electrolyte abnormalities. Singapore Med J 2012; 53:152-6.

13. Fromm MF, Kim RB, Stein CM, Wilkinson GR, Roden DM. Inhibition of P-glycoprotein-mediated drug transport: a unifying mechanism to explain the interaction between digoxin and quinidine. Circulation 1999; 99:552-7.

14. Eberl S, Renner B, Neubert A, et al. Role of p-glycoprotein inhibition for drug interactions: evidence from in vitro and pharmacoepidemiological studies. Clin Pharmacokinet 2007; 46:1039-49. 


\section{SINGAPORE MEDICAL COUNCIL CATEGORY 3B CME PROGRAMME} (Code SMJ 202001A)

Question 1. Chest radiograph features consistent with congestive heart failure include:
(a) Alveolar oedema (bat's wing opacities).
(b) Interstitial (Kerley B) lines.
(c) Cardiomegaly.
(d) Dilated upper lobe pulmonary vessels.

Question 2. Regarding digoxin:
(a) It is shown to have mortality benefits in patients with heart failure.
(b) It reduces the rate of hospitalisation in patients with heart failure.
(c) It is a first-line drug in the management of heart failure.
(d) It increases vagal tone for both sinoatrial and atrioventricular (AV) nodes.

Question 3. Electrocardiographic features of digoxin toxicity include:
(a) Reverse-tick ST segment.
(b) Atrial fibrillation.
(c) Bidirectional ventricular tachycardia.
(d) AV blocks.

Question 4. Symptoms that may suggest digoxin toxicity include:
(a) Palpitations.
(b) Confusion.
(c) Yellow-green disturbance of colour vision.
(d) Gastrointestinal symptoms (nausea, vomiting and diarrhoea).

Question 5. Drugs that are commonly associated with increased risk of digoxin toxicity include:
(a) Amiodarone.
(b) Erythromycin.
(c) Thyroxine.
(d) Verapamil.

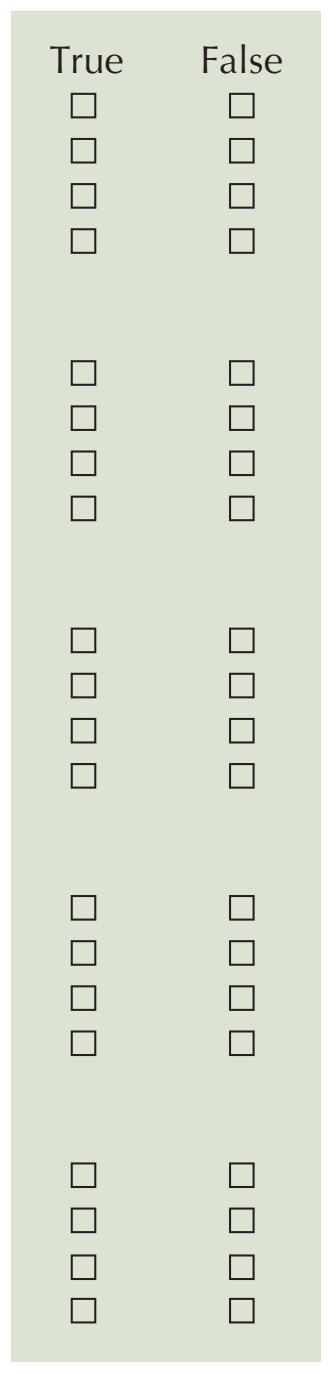

\section{Doctor's particulars:}

Name in full:

Specialty:

MCR no.:

Email:

\section{SUBMISSION INSTRUCTIONS:}

Visit the SMJ website: http://www.smj.org.sg/current-issue and select the appropriate quiz. You will be redirected to the SMA login page.

For SMA member: (1) Log in with your username and password (if you do not know your password, please click on 'Forgot your password?'). (2) Select your answers for each quiz and click 'Submit'.

For non-SMA member: (1) Create an SMJ CME account, or log in with your SMJ CME username and password (for returning users). (2) Make payment of SGD 21.40 (inclusive of $7 \%$ GST) via PayPal to access this month's quizzes. (3) Select your answers for each quiz and click 'Submit'.

RESULTS:

(1) Answers will be published online in the SMJ March 2020 issue. (2) The MCR numbers of successful candidates will be posted online at the SMJ website by 12 March 2020. (3) Passing mark is $60 \%$. No mark will be deducted for incorrect answers. (4) The SMJ editorial office will submit the list of successful candidates to the Singapore Medical Council. (5) One CME point is awarded for successful candidates. (6) SMC credits CME points according to the month of publication of the CME article (i.e. points awarded for a quiz published in the January 2020 issue will be credited for the month of January 2020, even if the deadline is in March 2020).

Deadline for submission: (January 2020 SMJ 3B CME programme): 12 noon, 5 March 2020. 